\title{
Acute Physical Stress Preconditions the Heart Against Ischemia/ Reperfusion Injury Through Activation of Sympathetic Nervous System
}

\author{
Alireza Imani, ${ }^{1,2}$ Hoda Parsa, ${ }^{1 *}$ Leila Gholami Chookalaei, ${ }^{1 *}$ Kamran Rakhshan, ${ }^{3}$ Masoomeh Golnazari, ${ }^{4}$ Mahdieh Faghihi \\ Department of physiology - School of Medicine - Tehran University of Medical Sciences, ${ }^{1}$ Tehran - Iran \\ Occupational Sleep Research Center - Baharloo Hospital - Tehran University of Medical Sciences, ${ }^{2}$ Tehran - Iran \\ Department of physiology - School of Medicine - Iran University of Medical Sciences, ${ }^{3}$ Tehran - Iran \\ Biology Department - Basic Sciences faculty - Hamedan Branch of Islamic Azad University, ${ }^{4}$ Hamedan - Iran \\ * Contributed equally to this work.
}

\begin{abstract}
Background: Stress is defined as a complicated state that related to homeostasis disturbances, over-activity of the sympathetic nervous system and hypothalamus-pituitary-adrenal axis responses. Cardiac preconditioning reduces myocardial damages.
\end{abstract}

Objective: This study was designed to assess the cardioprotective effects of acute physical stress against ischemia/ reperfusion $(I / R)$ injury through the activation of the sympathetic nervous system.

Methods: Thirty-two male Wistar rats were divided into four groups; (1) IR (n = 8): rats underwent I/R, (2) Acute stress $(\mathrm{St}+\mathrm{IR})(\mathrm{n}=\mathbf{8})$ : physical stress induced 1-hour before I/R, (3) Sympathectomy $(\operatorname{Symp}+\mathrm{IR})(\mathrm{n}=\mathbf{8})$ : chemical sympathectomy was done 24-hours before I/R and (4) Sympathectomy- physical stress $(\operatorname{Symp}+\mathrm{St}+\mathrm{IR})(\mathrm{n}=\mathbf{8})$ : chemical sympathectomy induced before physical stress and I/R. Chemical sympathectomy was performed using 6-hydroxydopamine $(100 \mathrm{mg} / \mathrm{kg}, \mathrm{sc})$. Then, the hearts isolated and located in the Langendorff apparatus to induce 30 minutes ischemia followed by $\mathbf{1 2 0}$ minutes reperfusion. The coronary flows, hemodynamic parameters, infarct size, corticosterone level in serum were investigated. $\mathrm{P}<0.05$ demonstrated significance.

Results: Physical stress prior to $\mathrm{I} / \mathrm{R}$ could improve left ventricular developed pressure (LVDP) and rate product pressure (RPP) of the heart respectively, $(63 \pm 2$ versus $42 \pm 1.2, p<0.05,70 \pm 2$ versus $43 \pm 2.6, p<0.05)$ and reduces infarct size $(22.16 \pm 1.3$ versus $32 \pm 1.4, p<0.05)$ when compared with the $I / R$ alone. Chemical sympathectomy before physical stress eliminated the protective effect of physical stress on I/R-induced cardiac damages (RPP: $21 \pm 6.6$ versus $63 \pm 2$, $p<0.01$ ) (LVDP: $38 \pm 4.5$ versus $43 \pm 2.6, p<0.01$ ) (infarct size: $35 \pm 3.1$ versus $22.16 \pm 1.3, p<0.01$ ).

Conclusion: Findings indicate that acute physical stress can act as a preconditional stimulator and probably, the presence of sympathetic nervous system is necessary. (Arq Bras Cardiol. 2019; 113(3):401-408)

Keywords: Stress, Mechanical; Sympathetic Nervous System; Hypothalamo-Hypophyseal System; Ischemia; Sympathectomy.

\section{Introduction}

Ischemic heart disease is the major health problem in the world. ${ }^{1}$ Although reperfusion, which refers to the rapid reestablishment of blood flow, can be one of the most effective methods against lethal injuries, ${ }^{2}$ it is associated with additional myocardial damage. ${ }^{3}$ Many methods have been proposed to diminish the deleterious effect of ischemia/reperfusion ( $\mathrm{I} / \mathrm{R})$ injuries and increase cardiac endurance. Based on these advances, induction the short-term episodes of $\mathrm{I} / \mathrm{R}$ or using the pharmacological agents earlier than prolonged $\mathrm{I} / \mathrm{R}$ period induces cardiac preconditioning which can successfully attenuate cellular necrosis and conserve high levels of energy.,

Mailing Address: Alireza Imani •

Department of Physiology - School of Medicine - Tehran University of Medical Sciences, Tehran - Iran

E-mail:aimani@tums.ac.ir

Manuscript received Auust 26, 2018, revised manuscript November 18, 2018, accepted October 16, 2018

DOI: 10.5935/abc.20190189
Sympathetic nervous system and hypothalamus-pituitaryadrenal (HPA) axis are two coordinated defence systems. They can mediate two-way brain-body communication during stressful situations. ${ }^{6}$ Autonomic system activation contributes to behavioral responses in animals and enables them to regulate homeostasis and improve endurance. ${ }^{7}$ Stress is characterized as a general HPA axis response against potential and deleterious stimuli. ${ }^{8}$ In fact, stress through increasing the activity of HPA axis and corticosterone release plays a critical role in coordinating of neuroendocrine, autonomic and behavioral functions and leads to adaptive responses. ${ }^{9,10}$ The activity of the sympathetic nervous system increases and neurotransmitter secretion alters during the occurrence of stress. ${ }^{11}$ Several body systems such as nervous, cardiovascular and immune systems are influenced by stress. Moreover, significant changes in hemodynamic parameters such as; heart rate (HR) and blood pressure are observed during stress which ultimately may lead to heart diseases. ${ }^{12}$ On the other hand, the release of norepinephrine from the sympathetic nervous system is increased during lethal ischemia and it has a role for inducing of $\mathrm{I} / \mathrm{R}$ injuries through the generation of hydroxyl free radicals. This current study was designed to evaluate the role of the sympathetic nervous system in mediating acute stress-induced cardioprotection against I/R injury in isolated rat heart. 


\section{Methods}

A total of 32 male Wistar rats (200-250g) were kept in an air-conditioned room on a 12 hours light-dark cycle, at $22 \pm 2{ }^{\circ} \mathrm{C}$, with free access to water and food. The experimental protocols followed in this study conformed to the Guidelines for the Care and Use of Laboratory Animals published by the National Institutes of Health (NIH Publication No. 85-23, revised 1996) and were further approved by the institutional ethical committee of Tehran University of Medical Sciences (Tehran, Iran).

Stress box apparatus was used for physical stress exposure. It contained stainless bars at the bottom, connected to electroshock device using a connecting cable. Physical stress was induced using electrical foot shock $(1 \mathrm{~mA})$ for 10 seconds with 50 seconds intervals for one hour. After that, animals were anaesthetized with sodium thiopental $(60 \mathrm{mg} / \mathrm{kg}$, i.p), ${ }^{13}$ put on a surgical board. The chest was opened and the surgical silk suture (6-0) placed under the root of the left anterior descending coronary artery (LAD). Finally, the heart was removed from the chest and connected to Langendorffperfusion apparatus. Heart was perfused in a retrograde manner using Krebs-Henseleit bicarbonate buffer (in mmol/l): sodium bicarbonate $=25$, sodium chloride $=118.5$, potassium chloride $=4.7$, magnesium sulfate $=1.2$, glucose $=11$, gassed with $95 \% \mathrm{O}_{2}$ and $5 \% \mathrm{CO}_{2}\left(\mathrm{pH}=7.3-7.4\right.$ at $\left.37^{\circ} \mathrm{C}\right)$. Thereafter, the ends of the suture were passed through a plastic tube to create a snare for ischemia induction. Reperfusion was performed by releasing the snare. Latex fluid- filled balloon was inserted inside the left ventricle and connected to a pressure transducer (Harvard, March-Hugsteten, Germany), the biolab apparatus was used for recording the ventricular pressures. During the surgical procedure, recording was done during three designated periods: 20-30 minutes of the baseline (a period without any manipulation), 30 minutes of the local ischemia and 120 minutes of the reperfusion. After reperfusion, LAD was occluded again; Evans Blue dye ( $3 \mathrm{~mL}$ of $1.5 \%$ solution) was administrated to discriminate ischemic zone (the area at risk; [AAR]) from non-ischemic zone. ${ }^{14} \mathrm{After}$ freezing $\left(-20^{\circ} \mathrm{C}\right.$ for 24 hours), heart tissue was sliced into $2 \mathrm{~mm}$ transverse sections and kept in 1\% 2, 3, 5 triphenyltetrazolium chloride (TTC in $0.1 \mathrm{M}$ phosphate buffer, $\mathrm{pH}=7.4$ Sigma) solution for 15-20 min at $37^{\circ} \mathrm{C}$ to delineate ischemic from infarct zone. ${ }^{15}$ At the end of the experiments, the ratio of AAR and infarcted size (IS) were calculated by the Photoshop program.

Animals were allotted in 4 groups:

1. IR group $(n=8)$ : Rats were kept in stress box device (without stress exposure) for 1 hour and then, hearts were removed from the chest and subjected to ischemia and reperfusion.

2. Acute stress $(S t+I R)$ group $(n=8)$ : Rats were exposed electrical feet shock in the stress box for 1 hour and then, hearts were removed from the chest and subjected to ischemia and reperfusion.

3. Sympathectomy (Symp+IR) group $(n=8)$ : chemical sympathectomy was done by injection of a 6-hydroxydopamine (6-OHDA, $100 \mathrm{mg} / \mathrm{kg}$, sc) 24 hours prior to $\mathrm{I} / \mathrm{R}$ induction. ${ }^{16}$
4. Sympathectomy- physical stress (Symp $+\mathrm{St}+\mathrm{IR})$ group $(n=8)$ : chemical sympathectomy was done 24 hours prior to physical stress and $\mathrm{I} / \mathrm{R}$ induction.

We measured serum corticoesterone levels by ELISA method. Moreover, systolic blood pressure was measured via non-invasive technique (Tail Cuff and power lab) to confirm chemical sympathectomy $(n=4)$.

\section{Statistical analysis}

The sample size and group divisions were defined based on our previous studies. ${ }^{17}$ All data are reported as means \pm S.E.M. Normality was checked using Kolmogorov-Smirnov test, SPSS software version 20. One way ANOVA and Tukey post hoc test was done for comparison of parameters between different groups. Analysis of changes in mean values over three times was done using repeated measurement ANOVA within each group. Sample t-test was used to compare systolic blood pressure before and after sympathectomy. Significant changes were considered as $\mathrm{p}<0.05$.

\section{Results}

Effect of acute physical stress on coronary flow and heart rate

Figure 1 shows coronary flow (CF) at the end of the baseline, ischemia and reperfusion periods. There are significant differences for $\mathrm{CF}$ at the end of ischemia and reperfusion when compared to the end of the baseline period within groups ( $p<0.01)$. HR was significantly decreased at the end of both ischemia and reperfusion in comparison with the end of baseline period within groups ( $p<0.01)$, but no significant change were observed between different groups (Figure 2).

\section{Effect of acute physical stress on cardiac} hemodynamic parameters

The left ventricular developed pressure (LVDP, the difference between intraventricular systolic and diastolic pressures), rate product pressure (RPP, LVDP multiplied by HR) and were diminished at the end of reperfusion in comparison to the end of baseline period among groups,

The amount of RPP and LVDP in acute stress group were extremely increased in comparison to IR group $(p<0.05$ in induction of chemical sympathectomy before physical stress were considerably decreased RPP and LVDP in comparison to physical stress group $p<0.05$, but there is no marked difference between sympathectomy group when compared to IR group (Figure 3).

\section{Effect of acute physical stress on infarcts size (\%IS/AAR)}

Figures 4 shows the size of the infarct (\%IS/AAR) in different groups.

Infarct size was greatly deacreased in acute stress group as compared to the IR group ( $p<0.05$ ), but there was no considerable change in chemical sympathectomy group as compared to the control group. Chemical sympathectomy prior to acute physical stress represented no extermly change 


\section{Original Article}

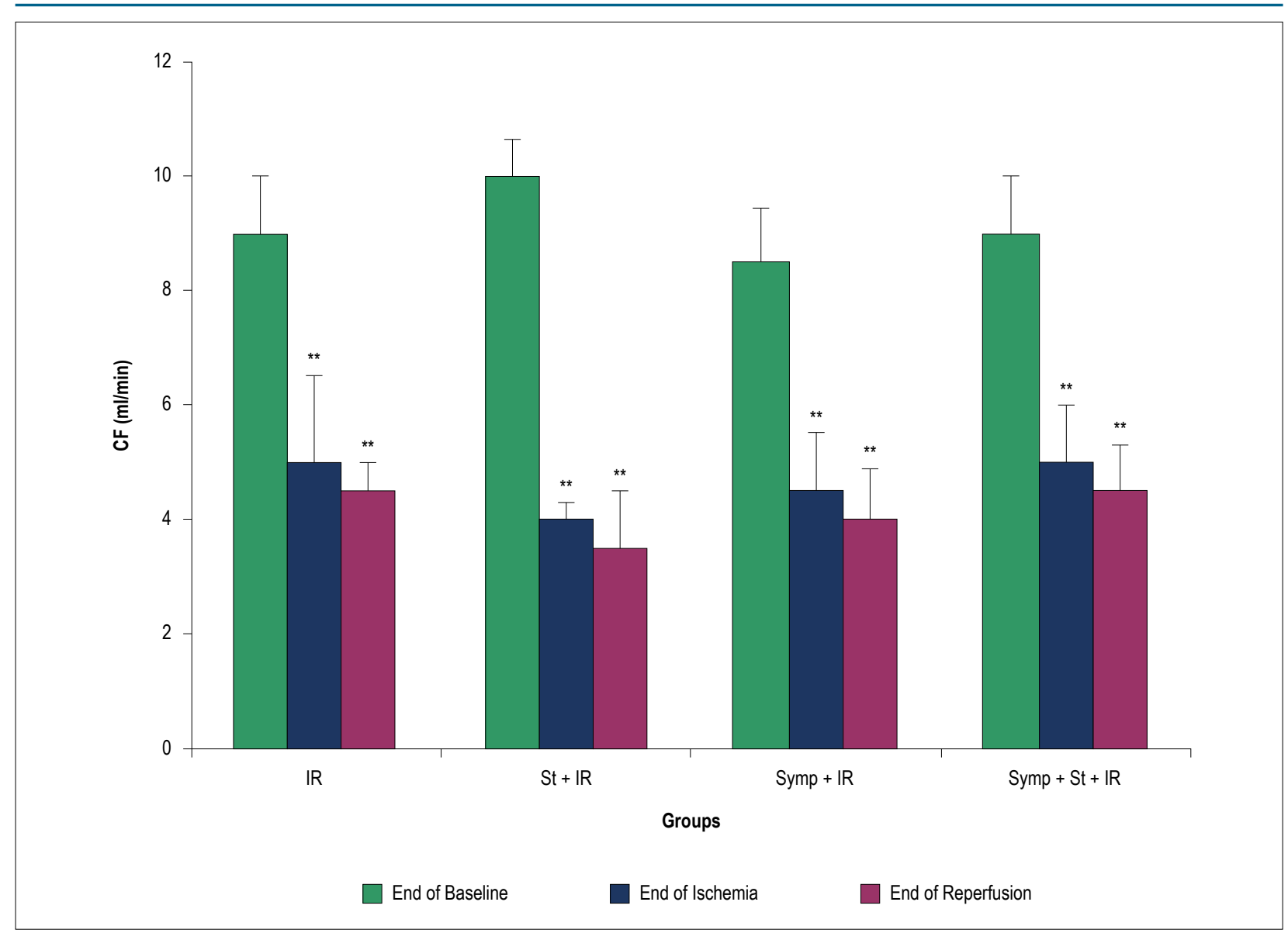

Figure 1 - Coronary flow (CF) at the end of baseline, ischemia and reperfusion periods. IR: Ischemia/reperfusion; St: Physical stress; Symp: Symapathectomy ${ }^{* *} p<0.01$ vs baseline phase within the same group.

as compared to IR group, while it has been shown significant reduced infarct size as compared with acute physical stress alone $(p<0.01)$.

\section{Effect of acute physical stress on corticosterone level in serum}

Figure 5 shows the serum level of corticosterone in different groups. Induction of acute physical stress without or with chemical sympathectomy in St and St+Symp+IR groups could increase the amount of serum corticosterone as compared to the IR group, $(p<0.01)$.

\section{Effect of chemical sympathectomy on Systolic blood pressure}

Figure 6 represents the significant reduction of systolic blood pressure after induction of chemical sympathectomy $(p<0.05)$.

\section{Discussion}

Nowadays daily life is associated with stress that is divided to acute stress and chronic stress, based on exposure duration. ${ }^{18}$ Acute stress mediates several neurogenic pathways. ${ }^{19}$ Electrophysiological recordings revealed that acute stress manifests good effects such as favour heightened arousal and increases cognitive flexibility in an attentional set- shifting task. ${ }^{20}$ In the other view, stress is divided into physical and psychological. A physical stressor such as surgery, trauma and heavy physical activity can trigger many cardiac events. ${ }^{21}$ Psychological stress can affect the cardiovascular system through metabolic, inflammatory and hormonal factors. ${ }^{22,23}$ In this study, we evaluated the effects of acute physical stress prior to sympathectomy on ischemia-reperfusion injuries in isolated rat heart.

\section{The effects of stress}

Our results showed that induction of acute stress prior to ischemia-reperfusion period led to a decrease in the infarct size, improve hemodynamic parameter and increase in the plasma corticosterone level as compared to IR group and Symp+IR group. Two paradoxical theories have been proposed to explain both advantage and disadvantage effect of stress on the heart. Extremely elevated $H R$, cardiac contractility and peripheral resistant due to exposure to acute stress can increase the cardiac load and oxygen consumption. In contrast, emerging evidences indicate the opposite effect, for example; cold restraint stress induces cardiac cell protection ${ }^{24}$ and it can diminish infarct size as a main parameter of cardiac damage. ${ }^{25}$ 


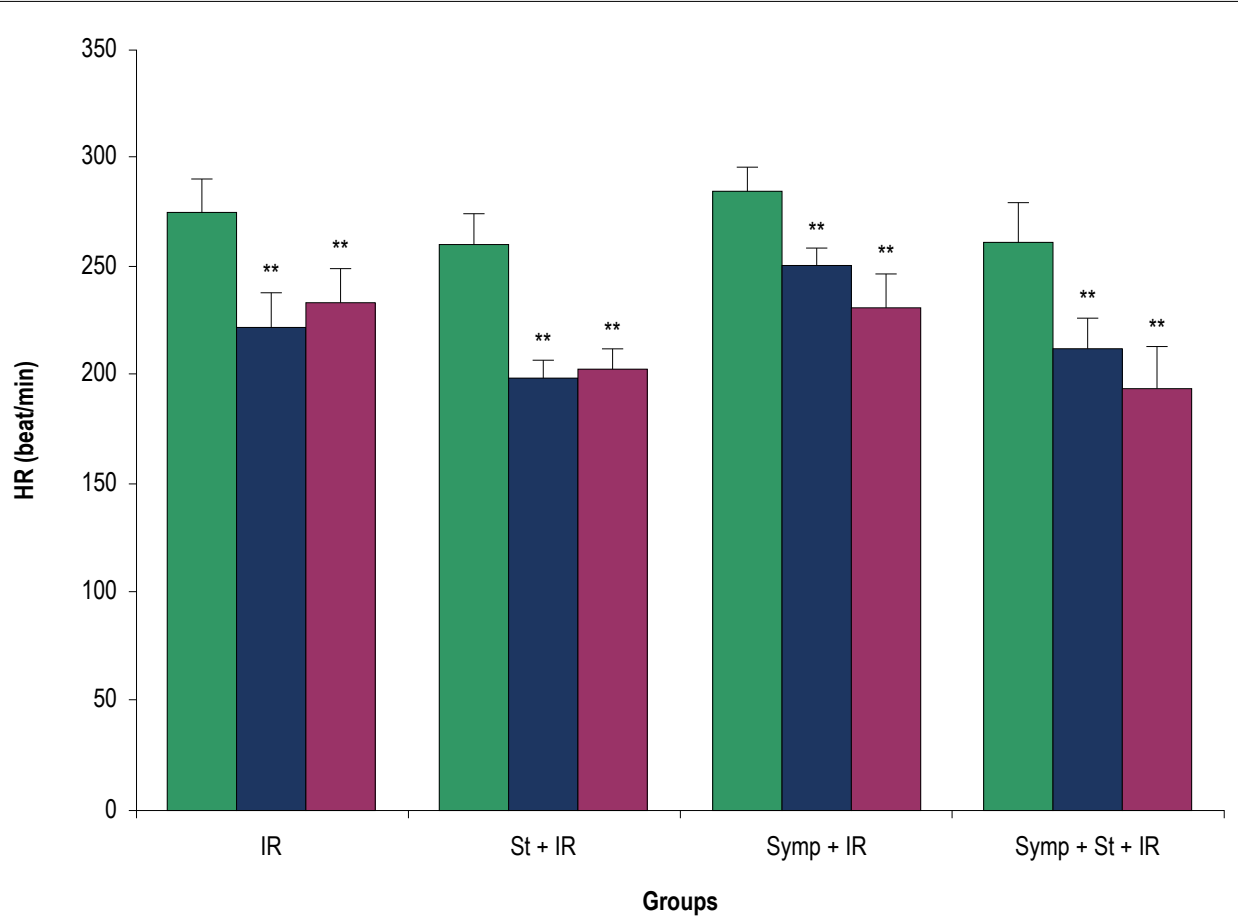

End of Baseline

End of Ischemia

End of Reperfusion

Figure 2 - Heart rate (HR) at the end of baseline, ischemia and reperfusion periods. IR: Ischemia/reperfusion; St: Physical stress; Symp: Symapathectomy. ${ }^{* *} p<0.01$ vs. baseline phase within the same group.

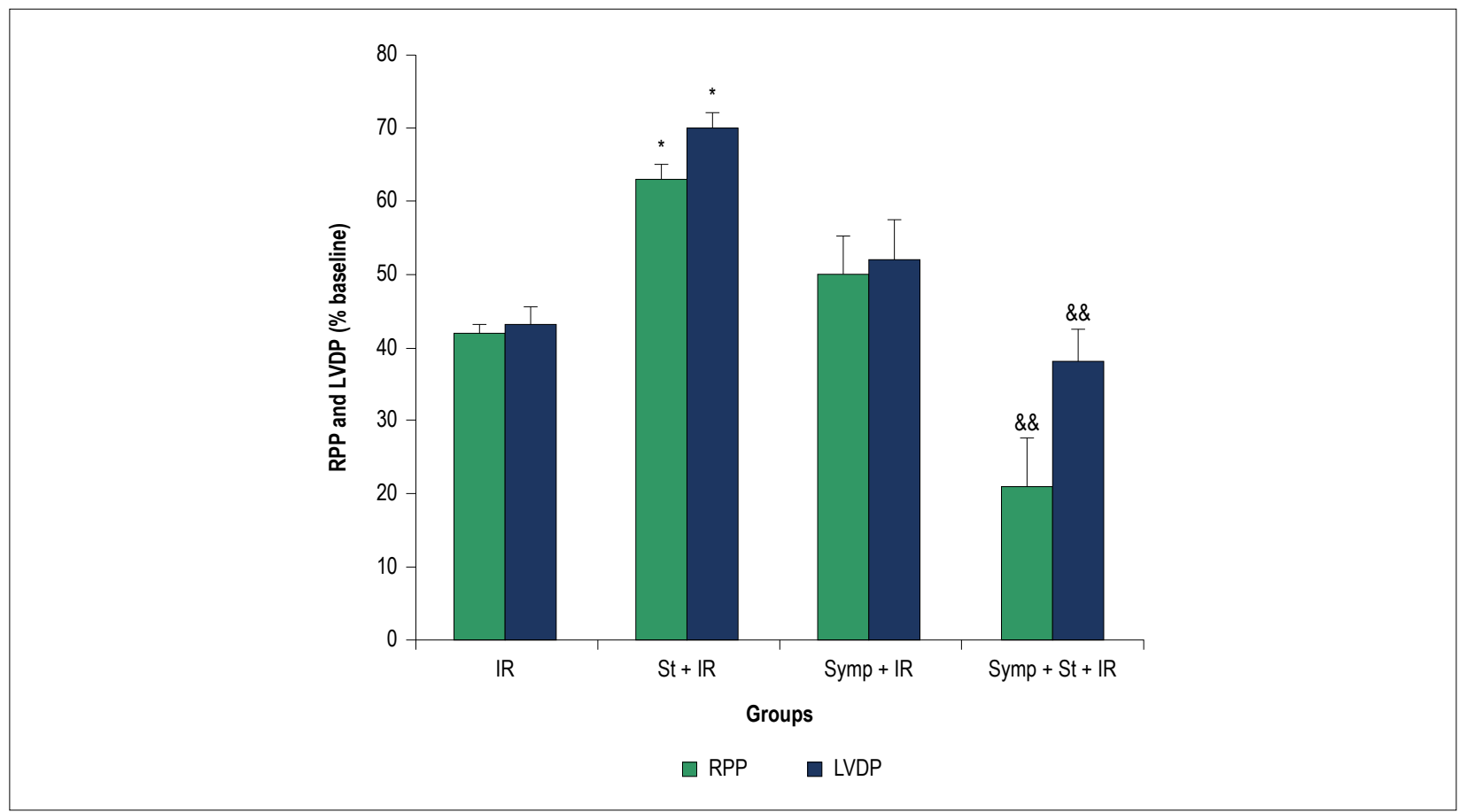

Figure 3 - Left Ventricular Developed Pressure (LVDP) and rate product pressure (RPP) at the end of reperfusion period. IR: Ischemia/reperfusion; St: Physical stress; Symp: Symapathectomy. ${ }^{*} p<0.05$ compared to $\mathbb{R}, \& \& p<0.01$ compared to $S t+\mathbb{R}$. 




Figure 4 - The percentage of infracts size (IS/AAR \%). IR: Ischemia/reperfusion; St: Physical stress; Symp: Symapathectomy. ${ }^{*} p<0.05$ compared to IR, \&\& $p<0.01$ compared to St+IR.



Figure 5 - Corticosterone level in serum. IR: Ischemia/reperfusion; St: Physical stress; Symp: Symapathectomy. ${ }^{* *} p<0.01$ compared to IR.

In this regard, Abe et al. demonstrated that acute stress attenuates ischemia-reperfusion injury in the kidney through the activation of sympathetic and anti-inflammatory pathway. ${ }^{26}$

Moreover, exposure to intermediate stress involves in cell protection against subsequent lethal ischemia, as a concept, preconditioning phenomenon..$^{27,28}$ It seems that acute physical stress exposure as a preconditioning agent protects the heart against I/R. We observed an increase in RPP and LVDP amounts due to acute stress induction in St+IR group as compared with IR group that indicates acute stress would trigger mechanisms to prepare the body for suitable responses to stimuli because the improvement of the cardiac function is important. Therefore, it seems that the effectiveness of stress induction is associated with; 1 . nature of the stressor, 2. stress episode duration, 3. intensity of the stimulus and 4 . stress predictability or unpredictability. In fact, each of the above factors effects on the neural and hormonal responses to stress. Our results showed that corticosterone is elevated after stress induction, and in Symp+St+IR group is higher than the IR group. It is well established that stress enhances the activity of the HPA axis which results to increase corticosterone secretion ${ }^{22,29}$ that can be protective as it prepares the organism to deal with challenges. Based on our results sympathectomy did not effect on stress-induced elevated corticosterone possibly because stress affects HPA axis through different mechanisms such as changes in metabolic and inflammatory factors in addition to increased sympathetic nervous system. ${ }^{22}$ Furthermore, this hormone induces changes in immune cells redistribution that enhance immune function. ${ }^{30}$ We showed that infarct size is decreased in St+IR group in comparison to the IR group and 


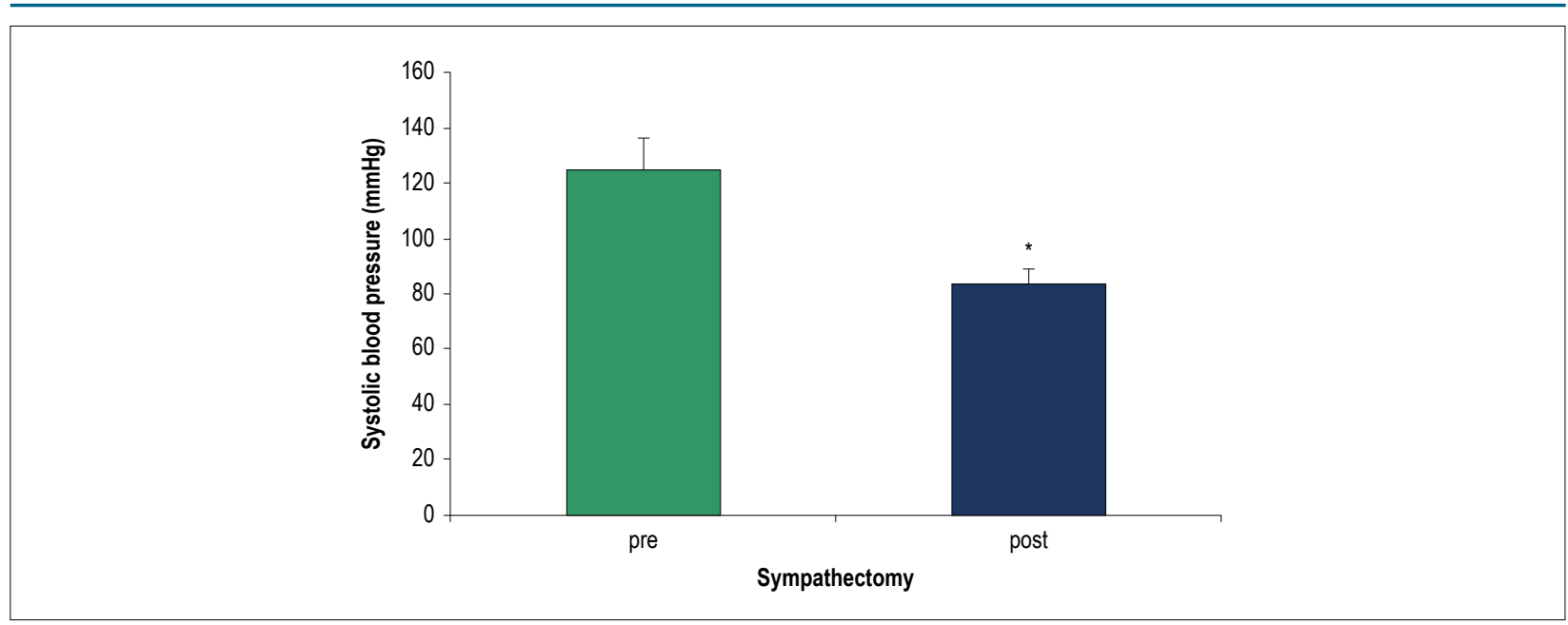

Figure 6 - Systolic blood pressure before and after chemical sympathectomy. ${ }^{*} p<0.05$ compared to before sympathectomy.

the hemodynamic parameters are improved in St+IR group in compare to the IR group. Diminished infarct size led to reducing in cardiac arrhythmia occurrence ${ }^{31,32}$ and also improved cardiac contractility. ${ }^{33}$ It seems the beneficial effects of acute stress induction may relate to the improvement of immune system function due to elevated corticosterone level encounter inflammatory factors, which trigger $\mathrm{I} / \mathrm{R}$ injuries.

\section{The effects of sympathectomy}

It has been established that exposure to stressful conditions increase autonomic nervous system activity. ${ }^{30}$ The cardioprotection of sympathetic activity has been investigated $^{34}$ and we used chemical sympathectomy after acute stress induction to confirm the protective effects of the sympathetic nervous system. Animals in Symp+IR group were subjected to chemical sympathectomy before induction of $\mathrm{I} / \mathrm{R}$ and there was no significant change in infarct size in comparison to IR group, indicating that chemical sympathetic denervation has no effect on IR injury. In addition, chemical sympathectomy prior to physical acute stress removed the cardioprotection effect of acute stress on infarct size in Symp + St+IR group that emphasizes the presence of sympathetic system is necessary for cardioprotective effects of acute stress. We found that acute stress induction after chemical sympathectomy could overcome harm effects of deleted sympathetic system on hemodynamic parameters in Symp +St+IR group when compared to Symp+IR group that indicates the essential role of sympathetic system physiological activity in regulating $\mathrm{HR}$, pressure and flow. ${ }^{35}$ Hara and Abiko declared norepinephrine has two opposite effects on ischemia damages according to the duration of ischemia, means that it could protect the heart with short ischemia and increase the injuries with prolonged ischemia. ${ }^{28}$ Positive and negative properties of the sympathetic nervous system are associated with the duration of stimulus exposure. At the long term ischemia, large quantities of norepinephrine are released from the sympathetic nervous system, acting as a source of the free radical and subsequent generation of $\mathrm{OH}$ free radical. ${ }^{36}$ Protective effect of norepinephrine can be emerged by producing energy for cardiac muscle in short term ischemia episode. ${ }^{37}$ Moreover, Yohimbine (as an alpha2 receptor antagonist) administration reduced the incidence of arrhythmia through increasing sympathetic norepinephrine release. ${ }^{37,38}$ According to our previous studies, pretreatment with alpha receptor agonist such as phenylephrine could protect the cardiomyocytes against I/R damages in isolated $H R{ }^{39}$ Activation of protein kinase-C (PKC) signalling pathway ${ }^{40}$ and $\mathrm{NO}$ release $\mathrm{e}^{41}$ by norepinephrine, are involved in the opening of mitochondrial KATP channels, which in turn can reduce mitochondrial calcium load ${ }^{42}$ and will lead to attenuation of norepinephrine beneficial effects. Also, we showed that systolic blood pressure declined after chemical sympathectomy that is compatible with this fact that muscle sympathetic outflow is responsible for the regulation of blood pressure. ${ }^{43}$ The limitations of this study were the method of induction physical stress does not commonly occur in daily life. Unfortunately, the data of corticosterone level in Symp+IR group were missed so we can't discuss the effect of sympathectomy on corticosterone level and comparison between $\mathrm{St}+\mathrm{IR}$ group and $\mathrm{Symp}+\mathrm{St}+\mathrm{IR}$ group is not significant. In consistence with our results, Lowrance et al. showed that stress-induced corticosterone level didn't change following pharmacological sympathectomy. ${ }^{44}$

\section{Conclusion}

The present study showed that induction of physical acute stress before I/R led to cardioprotection and chemical sympathectomy removed this beneficial effect of physical acute stress.

\section{Acknowledgements}

This study was supported by Tehran University of medical sciences.

\section{Author contributions}

Conception and design of the research: Imani A, Faghihi $M$; Acquisition of data: Rakhshan K, Golnazari M; Analysis and interpretation of the data: Imani A, Parsa H, Chookalaei LG, Faghihi M; Statistical analysis and Writing of the manuscript: 


\section{Original Article}

Imani A, Parsa H, Chookalaei LG; Critical revision of the manuscript for intellectual content: Imani A.

\section{Potential Conflict of Interest}

No potential conflict of interest relevant to this article was reported.

\section{Sources of Funding}

There were no external funding sources for this study.

\section{Study Association}

This article is part of the thesis of master submitted by Alireza Imani, from Tehran University of Medical Sciences.

\section{Ethics approval and consent to participate}

This study was approved by the Ethics Committee of the Tehran University of Medical Sciences under the protocol number 30486. All the procedures in this study were in accordance with the 1975 Helsinki Declaration, updated in 2013

\section{References}

1. Rochette L, Moreau D, Opie LH. Effect of repeated regional myocardial ischemia in the rat heart on reperfusion arrhythmias and release of norepinephrine. J Cardiovasc Pharmacol. 2001;38(1):78-89.

2. Gottlieb RA, Burleson KO, Kloner RA, Babior BM, Engler RL. Reperfusion injury induces apoptosis in rabbit cardiomyocytes. J Clin Invest. 1994;94(4):1621-8.

3. Lucchesi BR. Modulation of leukocyte-mediated myocardial reperfusion injury. Annu Rev Physiol. 1990;52:561-76.

4. Murry CE, Jennings RB, Reimer KA. Preconditioning with ischemia: a delay of lethal cell injury in ischemic myocardium. Circulation. 1986;74(5):1124-36

5. Anvari MA, Imani A, Faghihi M, Karimian SM, Moghimian M, Khansari M. The administration of oxytocin during early reperfusion, dose-dependently protects the isolated male rat heart against ischemia/reperfusion injury. Eur J Pharmacol. 2012;682(1-3):137-41.

6. Engelmann M, Landgraf R, Wotjak CT. The hypothalamic-neurohypophysial system regulates the hypothalamic-pituitary-adrenal axis under stress: an old concept revisited. Front Neuroendocrinol. 2004;25(3-4):132-49.

7. Parker VJ, Douglas AJ. Stress in early pregnancy: maternal neuro-endocrineimmune responses and effects. J Reprod Immunol. 2010;85(1):86-92.

8. Adam TC, Epel ES. Stress, eating and the reward system. Physiol Behav. 2007;91(4):449-58.

9. Angelucci L. The glucocorticoid hormone: from pedestal to dust and back. Eur J Pharmacol. 2000;405(1-3):139-47.

10. Korte SM. Corticosteroids in relation to fear, anxiety and psychopathology. Neurosci Biobehav Rev. 2001;25(2):117-42.

11. McEwen BS. Protective and damaging effects of stress mediators. N Engl J Med. 1998;338(3):171-9.

12. Lampert R, Jain D, Burg MM, Batsford WP, McPherson CA. Destabilizing effects of mental stress on ventricular arrhythmias in patients with implantable cardioverter-defibrillators. Circulation. 2000;101(2):158-64.

13. Todd MM, Chadwick H, Shapiro HM, Dunlop BJ, Marshall LF, Dueck R. The neurologic effects of thiopental therapy following experimental cardiac arrest in cats. Anesthesiology. 1982;57(2):76-86.

14. Mullane KM, Read N, Salmon JA, Moncada S. Role of leukocytes in acute myocardial infarction in anesthetized dogs: relationship to myocardial salvage by anti-inflammatory drugs. J Pharmacol Exp Ther. 1984;228(2):510-22.

15. Mello MT, Silva NPM. The use of triphenyltetrazolium chloride in the study of dehydrogenase activity of Brucellae. Mem Inst Oswaldo Cruz. 1955:53(1):45-58.

16. HeadrickJP. Ischemic preconditioning: bioenergetic and metabolic changes and the role of endogenous adenosine. J Mol Cell Cardiol. 1996;28(6):1227-40.

17. Choopani S, Imani A, Faghihi M, Askari S, Edalatyzadeh Z. chronic sleep deprevation and ventricular arrhythmias: effect of symphatic nervous system. J Cell Mol Anesthesia. 2016;1(2):56-61.

18. Wu S, Wong MC, Chen $\mathrm{M}$, Cho $\mathrm{CH}$, Wong TM. Role of opioid receptors in cardioprotection of cold-restraint stress and morphine. J Biomed Sci. 2004;11(6):726-31.

19. Hering D, Lachowska K, Schlaich M. Role of the sympathetic nervous system in stress-mediated cardiovascular disease. Curr Hypertens Rep. 2015;17(10):80.

20. Wood SK, Valentino RJ. The brain norepinephrine system, stress and cardiovascular vulnerability. Neurosci Biobehav Rev. 2017;74(PtB):393-400.

21. Moghimian M, Faghihi M, Karimian SM, Imani A. The effect of acute stress exposure on ischemia and reperfusion injury in rat heart: role of oxytocin. Stress. 2012;15(4):385-92.

22. Golbidi S, Frisbee JC, Laher I. Chronic stress impacts the cardiovascular system: animal models and clinical outcomes. Am J Physiol Heart Circ Physiol. 2015;308(12):H1476-98.

23. Hewagalamulage SD, Lee TK, Clarke IJ, Henry BA. Stress, cortisol, and obesity: a role for cortisol responsiveness in identifying individuals prone to obesity. Domest Anim Endocrinol. 2016;56(Suppl):S112-20.

24. Wilson TE, Crandall CG. Effect of thermal stress on cardiac function. Exerc Sport Sci Rev. 2011;39(1):12-7

25. Broadley K, Penson P. The roles of $\alpha$-and $\beta$-adrenoceptor stimulation in myocardial ischaemia. Auton Autacoid Pharmacol. 2004;24(4):87-93.

26. Abe $\mathrm{C}$, Inoue $\mathrm{T}$, Inglis MA, Viar KE, Huang L, Ye $\mathrm{H}$, et al. $\mathrm{C} 1$ neurons mediate a stress-induced anti-inflammatory reflex in mice. Nat Neurosci. 2017;20(5):700-7

27. Nonomura M, Nozawa T, Matsuki A, Nakadate T, Igarashi N, Kato B, et al. Ischemia-induced norepinephrine release, but not norepinephrine-derived free radicals, contributes to myocardial ischemia-reperfusion injury. Circ J. 2005;69(5):590-5.

28. Hara A, Abiko Y. Role of the sympathetic nervous system in the ischemic and reperfused heart. EXS. 1996;76:285-97.

29. Gong S, Miao Y-L, Jiao G-Z, Sun MJ, Li H, Lin J, et al. Dynamics and correlation of serum cortisol and corticosterone under different physiological or stressful conditions in mice. PloS One. 2015;10(2):e0117503.

30. Dhabhar FS. Effects of stress on immune function: the good, the bad, and the beautiful. Immunol Res. 2014;58(2-3):193-210.

31. Muscatell KA, Eisenberger NI. A social neuroscience perspective on stress and health. Soc Personal Psychol Compass. 2012;6(12):890-904.

32. Miller DB, $\mathrm{O}^{\prime}$ Callaghan JP. Neuroendocrine aspects of the response to stress. Metabolism. 2002;51(6 Suppl):5-10. 
33. Kario K, McEwen BS, Pickering TG. Disasters and the heart: a review of the effects of earthquake-induced stress on cardiovascular disease. Hypertens Res. 2003;26(5):355-67.

34. Abdul-Ghani S, Fleishman AN, Khaliulin I, Meloni M, Angelini GD, Suleiman $M$. Remote ischemic preconditioning triggers changes in autonomic nervous system activity: implications for cardioprotection. Physiol Rep. 2017;5(3):pii13085

35. Tobaldini E, Costantino G, Solbiati M, Cogliati C, Kara T, Nobili L, et al. Sleep, sleep deprivation, autonomic nervous system and cardiovascular diseases. Neurosci Biobehav Rev. 2017;74(Pt B):321-9.

36. Chrousos GP. Stress and disorders of the stress system. Nat Rev Endocrinol. 2009;5(7):374-81.

37. Heyndrickx GR, Vilaine JP, Moerman EJ, Leusen I. Role of prejunctional alpha 2-adrenergic receptors in the regulation of myocardial performance during exercise in conscious dogs. Circ Res. 1984;54(6):683-93.

38. Végh Á, Parratt JR. Noradrenaline, infused locally, reduces arrhythmia severity during coronary artery occlusion in anaesthetised dogs. Cardiovasc Res. 2002;55(1):53-63.
39. Naderi R, Imani A, Faghihi M, Moghimian M. Phenylephrine induces early and late cardioprotection through mitochondrial permeability transition pore in the isolated rat heart. J Surg Res. 2010;164(1):e37-42.

40. Tsuchida A, Liu Y, Liu GS, Cohen MV, Downey JM. alpha 1-adrenergic agonists precondition rabbit ischemic myocardium independent of adenosine by direct activation of protein kinase C. Circ Res. 1994;75(3):576-85.

41. Imani A, Faghihi M, Sadr SS, Niaraki SS, Alizadeh AM. Noradrenaline protects in vivo rat heart against infarction and ventricular arrhythmias via nitric oxide and reactive oxygen species. J Surg Res. 2011;169(1):9-15.

42. Imani A, Faghihi M, Sadr SS, Keshavarz M and Niaraki SS. Noradrenaline reduces ischemia-induced arrhythmia in anesthetized rats: involvement of $\alpha 1$-adrenoceptors and mitochondrial KATP channels. J Cardiovasc Electrophysiol. 2008;19(3):309-15.

43. Floras JS, Ponikowski P. The sympathetic/parasympathetic imbalance in heart failure with reduced ejection fraction. Eur Heart J. 2015;36(30):1974-82b.

44. Lowrance SA, lonadi A, McKay E, Douglas X and Johnson JD. Sympathetic nervous system contributes to enhanced corticosterone levels following chronic stress. Psychoneuroendocrinology. 2016 Jun;68:163-70. 